\title{
Estudo histológico e comparativo das papilas linguais dos cervídeos Mazama americana e Mazama gouzoubira por microscopia de luz e eletrônica de varredura ${ }^{1}$
}

\author{
Hanna Sibuya Kokubun ${ }^{2}$, Greyson V. Zanatta Esper ${ }^{3 *}$, André L. Rezende Franciolli ${ }^{3}$, \\ Fernanda Menezes de Oliveira e Silva ${ }^{3}$, Rose Eli G. Rici ${ }^{4}$ e Maria Angélica Miglino ${ }^{3}$
}

\begin{abstract}
Kokubun H.S., Esper G.V.Z., Franciolli A.L.R., Silva F.M.O., Rici R.E.G. \& Miglino M.A. 2012. [Histological and comparative analysis of lingual papillae of the deer Mazama americana and Mazama gouzoubira by light and scanning electron microscopy.] Estudo histológico e comparativo das papilas linguais dos cervídeos Mazama americana e Mazama gouzoubira por microscopia de luz e eletrônica de varredura. Pesquisa Veterinária Brasileira 32(10):1061-1066. Setor de Anatomia dos Animais Domésticos e Silvestres, Departamento de Cirurgia, Faculdade de Medicina Veterinária e Zootecnia, Universidade de São Paulo, Av. Prof. Orlando Marques de Paiva 87, São Paulo, SP 05508-270, Brazil. E-mail: greyson@usp.br

The type of feeding is dependent on the environment in which the animal lives, fact that's responsible for changes in morphology such as stratification, level of keratinization and functionality. Among the functional morphological structures of the tongue the papillae are worth mentioning due to their close relation to the diet. Two Cervidae species were used, five Mazama gouazoubira and two Mazama americana. Their tongues were divided into three parts, apex, body and root, and comparatively analyzed by light and scanning electron microscopy. The filiform, fungiform and vallate papillae were present in the two species' tongue and presented the same distribution, differing only in the quantity of vallate papillae on the root of the tongue, fact that might be related to the diet. Moreover, their distribution resembles that of other herbivore species.
\end{abstract}

INDEX TERMS: Tongue, Mazama americana, Mazama gouzoubira, ruminants, wild animals.

RESUMO.- 0 tipo de alimentação depende do ambiente em que o animal se encontra, sendo este um fator responsável pela alteração da morfologia, como a estratificação e o nível de queratinização da língua, e a funcionalidade da mesma. Dentre as estruturas morfológicas funcionais da língua, as papilas vêm merecendo destaque devido a sua estreita relação com a dieta. Foram utilizadas duas espécies de cervídeos: cinco $\mathrm{Ma}$ zama gouazoubira e duas Mazama americana, dividindo-se a língua em três partes: ápice, corpo e raiz. Analisou comparativamente a língua de duas espécies por meio de microscopia de

\footnotetext{
${ }^{1}$ Recebido em 11 de março de 2012.

Aceito para publicação em 20 de julho de 2012

${ }^{2}$ Aluna(o) de Graduação em Medicina Veterinária, Faculdade de Medicina Veterinária e Zootecnia (FMVZ), Universidade de São Paul (USP), Av. Prof. Orlando Marques de Paiva 87, Cidade Universitária, São Paulo, SP 05508-900, Brasil.

${ }^{3}$ Setor de Anatomia dos Animais Domésticos e Silvestres, Departamento de Cirurgia, FMVZ-USP, São Paulo, SP. *Autor para correspondência: greyson@usp.br

${ }^{4}$ Especialista em Laboratório do Setor de Anatomia dos Animais Domésticos e Silvestres, Departamento de Cirurgia, FMVZ-USP, São Paulo, SP.
}

luz e eletrônica de varredura. As papilas filiforme, fungiforme e valada apresentaram na língua das duas espécies estudadas, e com a mesma distribuição, mudando apenas a quantidade e formato de papilas filiformes no ápice lingual e a quantidade e disposição das papilas valadas na raiz da língua, fator este que pode ser ligado à dieta dos animais. . Além disto, sua distribuição é semelhante à de outras espécies de herbívoros.

TERMOS DE INDEXAÇÃO: Língua, Mazama americana, Mazama gouzoubira, ruminantes, animais silvestres.

\section{INTRODUÇÃo}

Os cervídeos são animais ruminantes e ungulados, divididos em 45 espécies, chamados comumente de veados e cervos. Tem distribuição geográfica extremamente ampla e está presente em toda América, Europa e Ásia (Walker 1991, Duarte 2006).

As espécies Mazama gouazoubira e Mazama americana da ordem Artiodactyla apresentam tamanhos médios de 50 e $65 \mathrm{~cm}$ e peso em torno de $18 \mathrm{~kg}$ e $30 \mathrm{~kg}$, respectivamente. Algumas espécies do gênero Mazama são frugívoras, e 
seu habitat se estende do sul do Uruguai ao norte do estado do Mato Grosso e da Cordilheira dos Andes até o Atlântico para a Mazama gouazoubira, enquanto a Mazama americana do México ao norte da Argentina (Duarte 2006, Abril et al. 2010, Ferreira et al. 2011).

A alimentação da espécie Mazama gouazoubira, localizada na região da Argentina, é composta basicamente de algumas forragens como dicotiledôneas, espécies lenhosas (arbustos e árvores), e ervas, não sendo encontrada a ingestão de gramíneas (Kufner et al. 2008). Em cativeiro, os cervídeos recebem, em geral, concentrados para bezerros, potros e cães, além de frutos e vegetais como gramíneas, leguminosas e verduras (Duarte 2006).

0 tipo de alimentação depende do ambiente em que o animal se encontra, sendo este um fator responsável pela alteração da morfologia, como a estratificação e o nível de queratinização da língua, e a funcionalidade da mesma. Como exemplo, a aquisição de um maior nível de estratificação e queratinização durante a transição do ambiente aquático para o ambiente terrestre, sendo estas mudanças as mais importantes no epitélio lingual. Nos mamíferos a queratinização está normalmente associada com a mucosa mastigatória oral que reveste a parte dorsal do corpo da língua e parece estar relacionada como uma maneira de resistir contra danos no tecido (Iwasaki 2002, Zheng \& Kobayashi 2006). Quanto ao nível de estratificação, observa-se que em animais com o epitélio lingual queratinizado apresentam uma camada além da lâmina própria, camada basal ou germinativa e camada granulosa, denominada estrato córneo (Benetti et al. 2009).

Devido às adaptações, como, condições ambientais, diversificação dietética e evolução das espécies, a língua pode apresentar uma variação morfológica com um papel importante na alimentação e na auto-limpeza (Iwasaki 2002). Dentre as estruturas morfológicas funcionais da língua, as papilas vêm merecendo destaque devido a sua estreita relação com a dieta. Em sua maioria, os mamíferos apresentam quatro tipos de papilas: filiformes, fungiformes, foliadas e valadas, que apresentam função mecânica de fricção, pouca função gustativa, grande função gustativa e lipase lingual, respectivamente. É comum a ausência de papilas foliadas em herbívoros, porém é observada sua presença em Tragulus javanicus (Agungpriyono et al. 1995). Makiyama et al. (1998) demonstraram que a morfologia do epitélio lingual varia entre as espécies animais, com uma única configuração externa representada pelos vários tipos de papilas. Na maioria das espécies de ruminantes é possível observar uma estrutura elíptica proeminente na parte caudal do corpo da língua, na sua porção dorsal, denominada torus lingual. Na presente estrutura observa-se a presença das papilas maiores, que facilitaria a compressão do alimento contra o palato durante os movimentos da mastigação (Habel 1986, Silva 1999).

Este estudo teve como objetivo analisar comparativamente a língua de duas espécies de veados (Mazama americana e Mazama gouzoubira) por meio de microscopia de luz e eletrônica de varredura, a fim de mostrar quais as papilas existentes e ver as possíveis diferenças entre as espécies estudadas.

\section{MATERIAL E MÉTODOS}

Foram utilizadas sete línguas das espécies de cervídeos, sendo, cinco Mazama gouazoubira e duas Mazama americana, dividindo-se esta peça em três partes: ápice, corpo e raiz da língua. Os animais utilizados foram os da coleção do Museu de Anatomia, localizado na Faculdade de Medicina Veterinária e Zootecnia da USP, sendo que já se encontravam conservados em solução de formaldeído $10 \%$.

Após a secção da língua, os fragmentos foram preparados para a microscopia de luz, sendo fixados em paraformaldeído à $4 \%$ após uma prévia lavagem com solução tampão de fosfato (PBS) a $0,1 \mathrm{M} \mathrm{pH} 7,4$. As amostras foram desidratadas em concentrações crescentes de etanol e, posteriormente diafanizadas em xilol e incluídas em parafina. Realizou-se secções de aproximadamente $5 \mu \mathrm{m}$ de espessura com o uso de micrótomo Leica RM-2065. As secções foram coradas com as colorações convencionais Hematoxilina-eosina (HE) e Tricromio de Masson.

Para a microscopia eletrônica de varredura, utilizou-se um fragmento de aproximadamente de $2 \mathrm{~cm}$ de cada parte da língua (ápice, corpo e raiz) de cada espécie, sendo fixados em parafor-

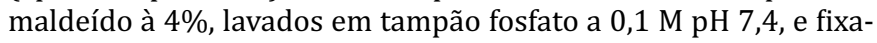
dos em tetróxido de ósmio a $1 \%$. Posteriormente foram desidratados a seco em ponto crítico (Balzers CPD 020) e colocados em suportes metálicos (stub) para metalização em ouro.

\section{RESULTADOS}

Através da vista macroscópica foi possível observar que a língua das duas espécies era recoberta por papilas. Estas papilas vistas na região do ápice eram papilas filiformes, com a adição de papilas fungiformes; no corpo havia a presença de papilas filiformes e fungiformes entremeadas, observando-se também no torus lingual na porção central desta região. Na raiz foi encontrada diferença entre as línguas das duas espécies em questão, nas laterais da língua de Mazama gouazoubira encontraram-se papilas valadas dispostas uma fileira no sentido rostro-caudal, enquanto na Mazama americana havia fileiras duplas de papilas valadas nas laterais da língua (Fig.1).

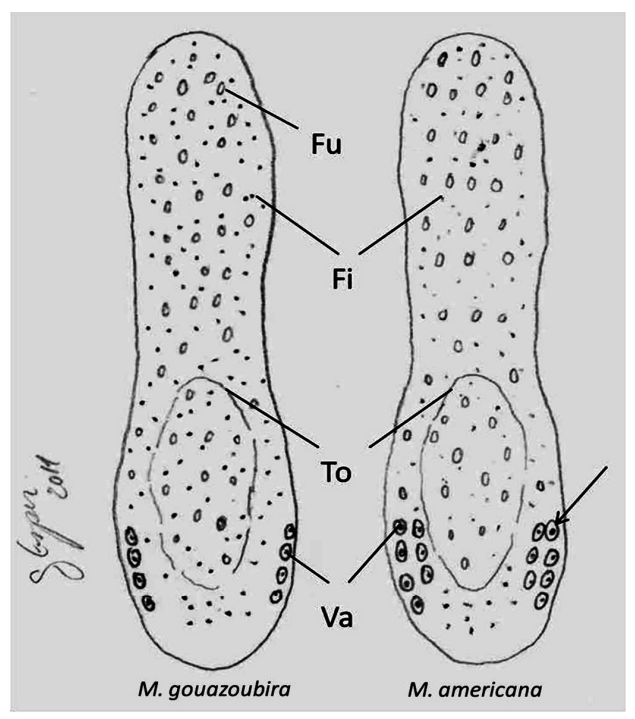

Fig.1. Esquema das línguas de Mazama. M. gouazoubira, com apenas uma fileira de papilas valadas. M. americana, com duas fileiras de papilas valadas (seta). (Fi) Papila filiforme, (Fu) Papila fungiforme, (Va) Papila valada, (To) Torus lingual. 
Através da microscopia de luz foi visto a mesma arquitetura observada na língua de ambas as espécies, com uma camada muscular composta de feixes entremeados de tecido conjuntivo e sobrepondo-se a esta a membrana mucosa, com revestimento da camada superficial composta de queratina recobrindo toda superfície da língua, inclusive as papilas linguais. As papilas filiformes estavam voltadas para a região caudal da língua, e visivelmente apresentavam maior quan- tidade de grânulos de querato-hialina que as demais papilas (Fig.2A,A'). No interior das papilas fungiformes observou-se corpúsculos gustativos desembocando em poros gustativos na superfície da papila e na superfície (Fig.2B,B') e lateral das papilas valadas de Mazama gouazoubira (Fig.2C,C'). Nas papilas gustativas foi possível observar feixes nervosos.

Quanto ao tamanho das papilas, foi observado que, em ambas as espécies, apresentam maior altura em relação à

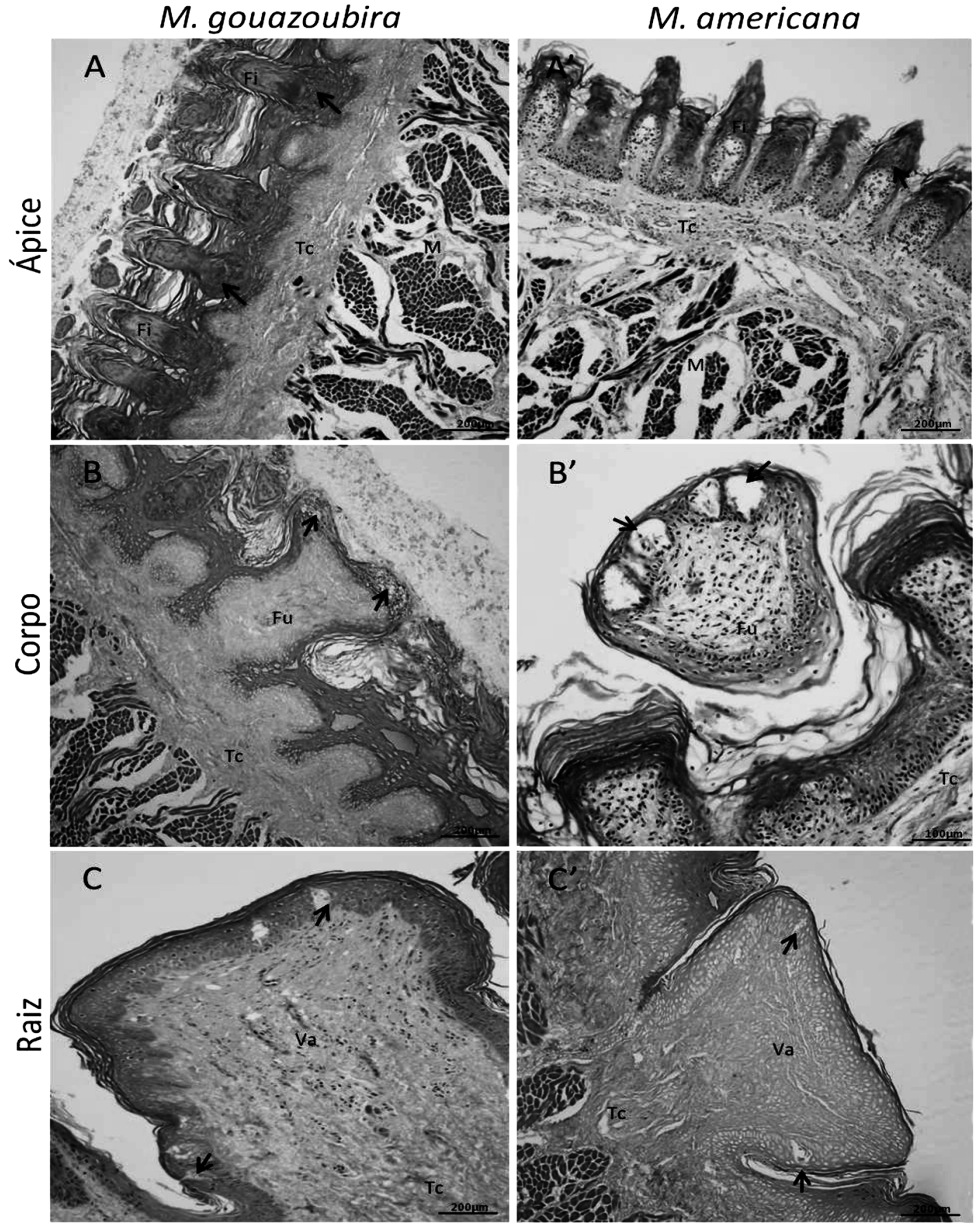

Fig.2. Regiões da língua de Mazama gouazoubira e M. americana. (A,A') Região de ápice lingual: observar papilas filiformes ( $\mathrm{Fi}$ ) compostas de queratina, originada de grânulos de querato-hialina (seta), tecido conjuntivo (Tc) e tecido muscular (M). (B,B') Região de corpo lingual: observar corpúsculos gustativos (setas) na superfície da papila fungiforme (Fu) e tecido conjuntivo (Tc). (C,C') Região de raiz lingual: observar o revestimento de queratina na superfície das papilas valada (Va), presença de botões gustativos na superfície e em sua lateral (seta). Colorações de Tricomio de Masson (A, B, C, C') e HE (A',B'). 
largura na porção rostral e conforme a porção caudal é alcançada, a altura das papilas diminui enquanto a largura aumenta.

Por microscopia eletrônica de varredura observou-se na língua da espécie Mazama gouazoubira dois tipos de papilas filiformes, com uma ampla distribuição e alta densidade do primeiro tipo de papila, sendo essa densidade maior nos dois primeiros terços da língua em que estas recobriram totalmente a superfície. Acompanhando-as rostro-caudalmente verifica-se que inicialmente há um tipo de papilas que apresentam comprimento longo com ponta arredondada e tornam-se outro tipo com pontas curtas e bordas quadradas, assemelhando-se a dedos e por vezes, quadrados, quando próximas ao torus lingual (Fig.3A). Ao se aproximar da raiz tornam-se novamente com bordas arredondados e com uma fissura, assemelhando-se a folhas (Fig.3A). As papilas fungiformes encontram-se dispostas entre as papilas filiformes, com superfície irregular, em pequena quantidade e bem distribuídas, sem alterações morfológicas evidentes (Fig.3B). A fileira única de papilas valadas, encontrada macroscopicamente, foi visualizada posteriormente em maior aumento pela microscopia eletrônica de varredura (Fig.3C').

Por microscopia eletrônica de varredura, observou-se no ápice da língua da Mazama americana uma combinação de papilas filiformes e fungiformes, estando à primeira em
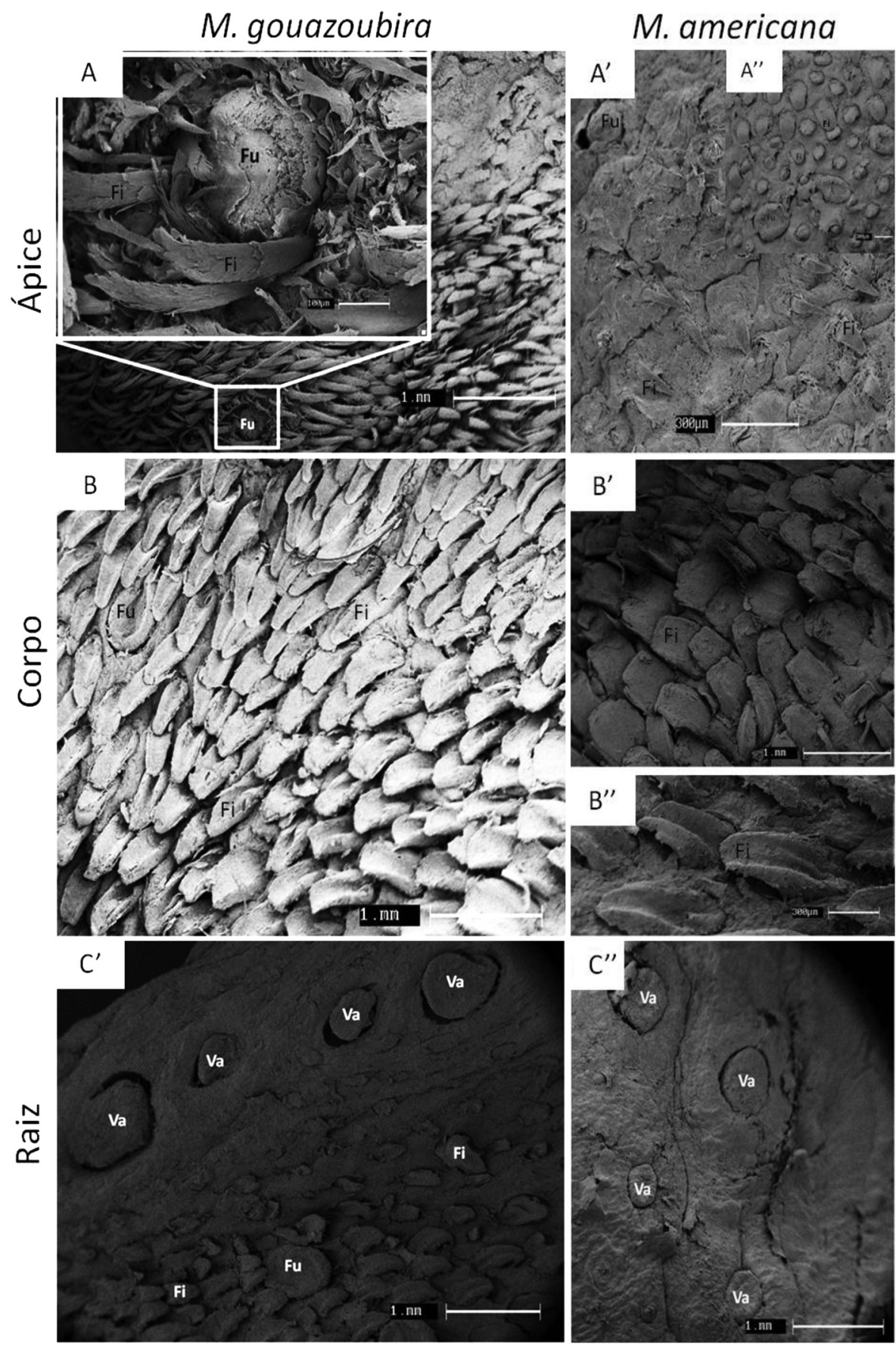

Fig.3. Eletromicrografias das regiões da língua de Mazama. gouazoubira e americana. (A, $\left.\mathbf{A}^{\prime}, \mathbf{A}^{\prime \prime}\right)$ Região de ápice lingual: (A) observar detalhe da papila fungiforme (Fu) localizada entre papilas filiformes (Fi) em formato de dedos. (A') observar papilas filiformes $(\mathrm{Fi})$ pequenas, afiladas e pontiagudas entremeadas por papilas fungiformes $(\mathrm{Fu})$. ( $\left.\mathrm{A}^{\prime \prime}\right)$ observar papilas filiformes (Fi) rasas e largas entremeadas por papilas fungiformes (Fu). Notar as diferenças morfológicas das papilas filiformes. (B,B',B') Região de corpo lingual: (B) observar papilas filiformes (Fi) e papilas fungiformes (Fu). (B') papilas filiformes com formato quadrado e curto. (B") observar papilas filiformes com formato de folha. (C e C') Região de raiz lingual: (C) observar fileira única de papilas valadas (Va), além de papilas filiformes (Fi) e papilas fungiformes (Fu). Notar que as papilas valadas são maiores quando comparadas com as papilas fungiformes. ( $C^{\prime}$ ) observar fileiras duplas verticalmente de papilas valadas (Va). 
maior quantidade em relação à segunda, e bem distribuída, apresentando baixa densidade e tamanho, afiladas e pontiagudas, sempre se voltando para a porção caudal da língua (Fig.3A', $A^{\prime \prime}$ ). Na região de transição do corpo para a raiz, observou-se no torus lingual papilas filiformes arredondadas, aparentando ser rasas e de formato mais arredondado, verifica-se uma menor quantidade das papilas fungiformes e entremeadas as filiformes (Fig. $3 \mathrm{~B}^{\prime}, \mathrm{B}^{\prime \prime}$ ). Na base lateral da raiz, em sua porção mais caudal existem papilas valadas com poros gustativos à mostra e dispostas em duas fileiras direcionadas rostro-caudalmente (Fig.3C").

\section{DISCUSSÃO}

Os resultados obtidos demonstraram as características da superfície dorsal da língua das espécies Mazama gouazoubira e Mazama americana. A superfície demonstrou papilas distribuídas por toda superfície em ambas as espécies, existindo diferenças quanto à morfologia e densidade, e tamanho das papilas entre regiões da língua, onde aparentam ser proporcionalmente maiores na M. gouazoubira que na $M$. americana quando comparadas ao tamanho da língua, fato este ligado ao tipo de alimentação que cada espécie analisada possui segundo Zheng \& Kobayashi (2006).

No ápice, as papilas filiformes de Mazama gouazoubira são maiores quando comparadas com as da Mazama americana por ter bordas levemente arredondadas e serem mais largas na base e apresentam maior altura, como visto na língua de bovinos (Steflik et al. 1983) e gado Zavot (Sari et al. 2010), enquanto na segunda espécie, serem mais finas e alongadas, semelhante a encontrada na língua de cabras Saanen (Kurtul \& Atalgin 2008, Fonseca et al. 2011), Camelus dromedarius (Qayyum et al. 1988), Hystrix cristata (Karan et al. 2011), Equus asinus (Abd-Elnaeim et al. 2002), Muntiacus reevesi (Zheng \& Kobayashi 2006) e de Oryctolagus cuniculus (Silva 1999).

Outro ponto levantado em nosso estudo refere-se às papilas localizadas na região de ápice estarem voltadas para a porção caudal da língua e terem em sua constituição histológica uma alta quantidade de grânulos de querato-hialina. Tal fato pode ser associado à função mecânica dessas papilas na língua (Dyce et al. 2004), e a queratinização ser devido a própria alimentação que ao friccionar as papilas ocorre um desgaste, fazendo com que aconteça um reposição normal desta queratina que tem intuito de proteger a mucosa oral (Bacha \& Bacha 2000).

Ainda nesta porção da língua, as papilas fungiformes são distribuídas amplamente, em pouco número e destacando-se, com sua superfície arredonda, conforme estas papilas em bovinos (Steflik et al. 1983), gado Zavot (Sari et al. 2010), Equus asinus (Abd-Elnaeim et al. 2002), Camelus dromedarius (Qayyum et al. 1988), Muntiacus reevesi (Zheng \& Kobayashi 2006), Oryctolagus cuniculus (Silva 1999) e ratos Wistar (Makiyama et al. 1998), porém ambas as espécies estudadas não apresentam superfície lisa e regular, mas sim uma superfície irregular com uma leve depressão no centro da papila como na língua de ratos Wistar (Makiyama et al. 1998) e Oryctolagus cuniculus (Silva 1999), diferentemente da cabra Saanen em que a forma da papila é de cogumelo, arredondada e convexa (Kurtul
\& Atalgin 2008). Outro ponto que deve ser levado em consideração, esta relacionado as espécies possuírem poros gustativos na superfície dorsal da papila fungiforme, proveniente de corpúsculos gustativos e garantindo às papilas a função de gustação, como visto em animais domésticos (Bacha \& Bacha 2000, Dyce et al. 2004).

No início do corpo da língua, os indivíduos Mazama gouazoubira apresentavam as papilas filiformes nas mesmas dimensões que na porção rostral e ao atingir a região do torus lingual, essas diminuíam de tamanho, assim como em Mazama americana, como visto no gado Zavot (Sari et al. 2010). Porém as papilas nesta região apresentaram bordas mais arredondadas, tornando-se, por vezes, levemente quadradas, assemelhando-se a dedos, como na língua de Hystrix cristata (Karan et al. 2011) e Oryctolagus cuniculus, porém com bifurcações ou trifurcações nesta ultima espécie (Silva 1999). Em cabras Saanen há grande número destas papilas, distribuídas randomicamente (Kurtul e Atalgin, 2008) como nas espécies estudadas; em Equus asinus as papilas filiformes são mais finas, longas e abundantes (Abd-Elnaeim et al. 2002) e suavemente mais finas em Muntiacus reevesi (Zheng \& Kobayashi 2006). As papilas fungiformes em ambas as espécies do gênero Mazama, no gado Zavot (Sari et al, 2010), nas cabras Saanen (Kurtul \& Atalgin 2008), no Camelus dromedarius (Qayyum et al. 1988), no Muntiacus reevesi (Zheng \& Kobayashi 2006) e Didelphis marsupialis (Mançanares et al. 2012) apresentaram-se distribuídas entre as papilas filiformes, tendo a mesma distribuição do ápice lingual.

Na transição destas duas regiões destaca-se no torus lingual, uma proeminência na base da língua presente nos cervídeos Mazama estudados, bem como visto em Muntiacus reevesi (Zheng \& Kobayashi 2006), bovinos (Steflik et al. 1983), gado Zavot (Sari et al. 2010), Camelus dromedarius (Qayyum et al. 1988), Oryctolagus cuniculus (Silva 1999), ratos Wistar (Makiyama et al 1998) e cabras Saanen (Kurtul \& Atalgin 2008). E em seguida temos a região da raiz da língua, que se destaca principalmente a disposição bilateral das papilas valadas na porção caudal da língua na qual está disposta em uma única fileira em Mazama gouazoubira, Muntiacus reevesi (Zheng \& Kobayashi, 2006) e Equus caballus (Guimarães et al. 2006); e disposta em duas fileiras paralelas em Mazama americana, cabras Saanen (Kurtul \& Atalgin 2008); diferentemente da disposição das papilas em Oryctolagus cuniculus, Equus asinus e Dasypus novemcinctus em que apresentam apenas um par destas papilas (Morais et al. 1994, Silva 1999, Abd-Elnaeim et al. 2002), três papilas em Callithrix penicillata (Branco et al. 2012) e somente uma papila central na língua de ratos Wistar Wistar (Makiyama et al. 1998). Cabe ressaltar que nas espécies Mazama gouazoubira e Mazama americana, juntamente com todas as outras espécies comparadas apresentaram poros gustativos desembocando nas paredes laterais da papila em direção à fenda que contorna as papilas, com exceção da Bradypus torquatus em que os poros gustativos encontravam-se na superfície da papila, indicam que a papila apresenta função gustativa, além da secreção de substâncias serosas pelas glândulas que auxiliam na limpeza da língua (Benetti et al. 2009). 


\section{CONCLUSÃO}

Os resultados deste estudo permitem concluir que as papilas se mostraram presentes na língua das espécies estudadas, e apresentaram a mesma distribuição, mudando apenas a quantidade e formato de papilas filiformes no ápice lingual e a quantidade e disposição das papilas valadas na raiz da língua, em ambas as espécies estudadas, fator este ligado a dieta em que os animais eram submetidos e sua distribuição semelhante as de outras espécies de herbívoros.

\section{REFERÊNCIAS}

Abd-Elnaeim M.M.M., Zayed A.E. \& Leiser R. 2002. Morphological characteristics of the tongue and its papillae in the donkey (Equus aisnus): A light and scanning electron microscopical study. Ann. Anat. 184:473480.

Abril V.V., Carnelossi E.A.G., González S. \& Duarte J.M.B. 2010. Elucidating the evolution of the red brocket deer Mazama Americana complex (Artiodactyla; Cervidae). Cytogenetic and Genome Research 128:177-187.

Agungpriyono S., Yamada J., Kitamura N., Nisa C., Sigit K. \& Yamamoto Y. 1995. Morphology of the dorsal lingual papillae in the lesser mouse deer, Tragulus javanicus. J. Anat. 187:635-640.

Bacha W.J. \& Bacha L.M. 2000. Color Atlas of Veterinary Histology. $2^{\text {nd }} e d$. Lippincott Williams and Wilkins, Philadelphia. 318p.

Benetti E.J., Pícoli L.C., Guimarães J.P., Motoyama A.A., Miglino M.A. \& Watanabe I.S. 2009. Characteristics of filiform, fungiform and vallate papillae and surface of inferface epithelium-connective tissue of the maned sloth tongue mucosa (Bradypus torquatus, Iliger 1811): Ligth and Scannig Electron Microscopy study. Anat. Histol. Embryol. 38(1):42-48.

Branco E., Pereira W.L., de Lima A.R., Franciolli A.L.R., Rici R.E., Miglino M.A., Muniz J.A. \& Imbeloni A. 2012. Ultrastructural aspects of Callithrix penicillata lingual papillae. Microsc. Res. Tech. 75(3):282-284.

Duarte J.M. 2006. Artiodactyla-Cervidae (Veado-catingueiro, Veado-campeiro, Cervo-do-pantanal), p.643-649. In: Cubas Z.S., Silva J.C.R. \& Catão-Dias J.L. (Eds), Tratado de Animais Selvagens. Roca, São Paulo.

Dyce K.M., Sack W.O. \& Wensing C.J.G. 2004. Tratado de Anatomia Veterinária. $3^{\underline{a}}$ ed. Saunders Elsevier, Philadelphia. 872p.

Ferreira A.O., Morini A.C., Favaron P.O., Passos C.C., Campos D.B., Miglino M.A. \& Guerra R.R. 2011. Avaliação morfológica das membranas fetais e da placenta de Mazama gouazoubira (veado-catingueiro) de vida livre no terço inicial da gestação. Pesq. Vet. Bras. 31(7):631-635.

Fonseca E.T., Oliveira C.M., Franciolli A.L.R. \& Miglino M.A. 2011. Características das papilas do dorso da língua de cabras (Capra hircus): estudo por meio de microscopia eletrônica de varredura e luz. Pesq. Vet. Bras. 31(Supl.1):67-73.
Habel R.E. 1981. Sistema digestivo dos ruminantes, p.852-856. In: Getty R. (Ed.), Anatomia dos Animais Domésticos. 5a ed. Interamericana, Rio de Janeiro.

Iwasaki S. 2002. Evolution of the structure and function of the vertebrate tongue. J. Anat. 201(1):1-13.

Karan M., Yilmaz S. \& Aydin A. 2011. Morphology of the filiform lingual papillae in porcupine (Hystrix cristata). J. Vet. Med. Anat. Histol. Embryol. 40(2):100-103

Kurtul I. \& Atalgin S.H. 2008. Scanning electron microscopic study on the structure of the lingual papillae of the Saanen goat. Small Rumin, Res. 80(1):52-56.

Makiyama M.C.K., Watanabe I.S., Mizusaki C.I. \& Konig Júnior B. 1998. Three-dimensional angioarchitecture of tongue corrosion casts from normal Young rats. Ann. Anat. 180(4):327-330.

Mançanares C.A., Santos A.C., Piemonte M.V., Vasconcelos B.G., Carvalho A.F., Miglino M.A., Ambrósio C.E. \& Neto A.C. 2012. Macroscopic and microscopic analysis of the tongue of the commom opossum (Didelphis marsupialis). Microsc. Res. Tech., May 12, 2012. DOI: 10.1002/jemt.22070.

Martinez M., Martinez F.E., Pinheiro P.F.F., Almeida C.D.C., Segatelli T.M. \& Watanabe I. 2000. Estudio al microscópicos electronico de barrido de la de lengua chinchila (Chinchilla laniger). Chil. Anat. 18(1). Disponível em <http://www.scielo.cl/scielo,php/script=sci_arttext\$pid=S0716$-98682000000100007 \& \operatorname{lng}=e s \& n r m=i s o>$ Acessado em 27 jan. 2011. DOI: $10.4067 / \mathrm{S} 0716-98682000000100007$.

Morais J.O.R., Watanabe I.S. \& Koning Júnior B. 1994. Scanning electron microscopy of the lingual mucosa of the nine-banded armadillo Dasypus novemcintus. Ann. Anat. 176(4):357-361.

Qayyum M.A., Fatani J.A. \& Mohajir A.M. 1988. Scanning electron microscopic study of the lingual papillae of the one humped camel, Camelus dromedarius. J. Anat. 160:21-26.

Sari E.K., Harem M.K. \& Harem I.S. 2010. Characteristics of dorsal lingual papillae of Zavot cattle. J. Anim. Vet. Adv. 9(1):123-130.

Silva M.C.P. 1999. Características morfológicas da superfície de interface epitélio-tecido conjuntivo da mucosa lingual de coelhos (Oryctolagus cuniculus): estudo aos microscópios de luz e eletrônico de varredura. Dissertação de Mestrado em Ciências Biológicas, Instituto de Ciências Biomédicas, Universidade de São Paulo, SP. 100p.

Steflik D.E., Singh B.B., Mckinney R.V. \& Boshell J.L. 1983. Correlated TEM, SEM, and histological observations of filiform papillae of the cow tongue. Acta Anat. 117:21-30.

Walker E. 1991 Mammals of the World. John HopkinsUniversty Press, Baltimore.1398p.

Zheng J. \& Kobayashi K. 2006. Comparative morphological study on the lingual papillae and their connective tissue cores (CTC) in reeves' mutjac deer (Muntiacus reevesi). Ann. Anat. 188:555-565. 